Recherches en histoire de l'art, histoire des civilisations, archéologie, anthropologie et muséologie

$13 \mid 2019$

Cahiers 13

\title{
John McHale, l'Amérique passée à la machine
}

John McHale, America through the machine

\section{Juliette Bessette}

\section{QpenEdition \\ Journals}

Édition électronique

URL : http://journals.openedition.org/cel/1875

DOI : $10.4000 /$ cel. 1875

ISSN : 2262-208X

Éditeur

École du Louvre

\section{Référence électronique}

Juliette Bessette, « John McHale, l'Amérique passée à la machine », Les Cahiers de l'École du Louvre [En ligne], 13 | 2019, mis en ligne le 17 juin 2019, consulté le 17 septembre 2019. URL : http:// journals.openedition.org/cel/1875; DOI : 10.4000/cel.1875

Ce document a été généré automatiquement le 17 septembre 2019.

\section{(c) $($ i) $(9)$}

Les Cahiers de l'École du Louvre sont mis à disposition selon les termes de la licence Creative Commons Attribution - Pas d'Utilisation Commerciale - Pas de Modification 4.0 International. 


\section{John McHale, l’Amérique passée à la machine}

John McHale, America through the machine

Juliette Bessette

NOTE DE L'AUTEUR

Laboratoire : Sorbonne Université, Centre André Chastel (UMR 8150), Galerie Colbert, 2 rue Vivienne, 75002 Paris. 
Fig. 1 et Fig. 2

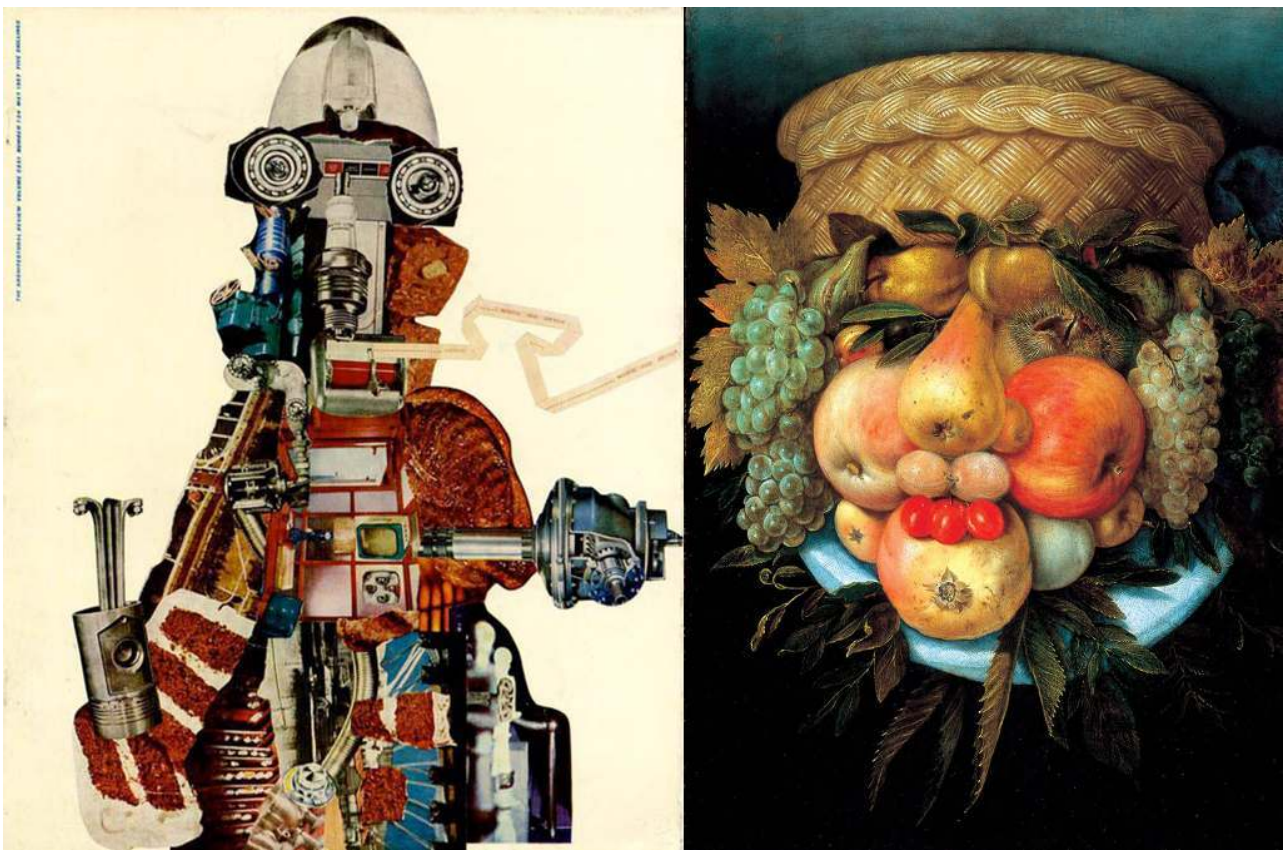

À gauche : John McHale, Machine Made, America, 1956, collage original non localisé/perdu, dimensions inconnues, reproduit en couverture du magazine Architectural Review, mai 1957.

(C) Architectural Review

À droite : Guiseppe Arcimboldo, Tête réversible avec panier de fruits, vers 1590, peinture à l'huile sur bois, $56 \times 42 \mathrm{~cm}$, French \& Company, New York

(c) French \& Company, New York

1 Se détachant sur fond crème, une figure humanoïde (fig. 1) dévisage le spectateur. Son corps est constitué de composants mécaniques, principalement des pièces détachées d'automobiles : les yeux sont faits de jantes, le bras gauche est un piston, le bras droit un joint universel, le nez une bougie d'allumage et le cerveau, une ampoule de phare. La puissance visuelle de cette image provient du détournement du sens initial des éléments iconographiques, formant une nouvelle composition. Ce personnage est issu du même procédé paréidolique que celui utilisé par le peintre de la Renaissance italienne Giuseppe Arcimboldo (fig. 2), mais les éléments hétéroclites qui le composent ne sont pas peints : ce sont des fragments imprimés, découpés et soigneusement assemblés par la technique du collage, qui les laisse assez lisibles. Il a été composé par l'artiste britannique John $\mathrm{McHale}^{1}$ dans les années 1950, au sein d'une série d'œuvres que son ami le critique d'art et théoricien Lawrence Alloway décrit comme constituées des « matériaux jetables de notre culture imprimée ${ }^{2}$ » :

Il utilisait des reproductions en couleur sur papier glacé prises dans des magazines à grand tirage. Ses silhouettes étaient en quelque sorte démocratiquement arcimboldesques, mais, alors que les personnages d'Arcimboldo étaient composés avec les outils propres à leurs métiers ou avec leurs attributs saisonniers, ceux de McHale étaient des portraits de consommateurs, abondamment nantis de toutes les bonnes choses qu'offrait l'après-guerre ${ }^{3}$.

Des images de nourriture cohabitent ici singulièrement avec l'environnement mécanique évoqué plus haut: steak haché en guise d'épaule, biceps de gâteau au chocolat. À la place du cœur, se trouve un écran de télévision. Les images de McHale sont « définies par ce qu'elles consomment [...] en cela, elles sont aussi éphémères que 
les produits de consommation desquels elles sont constituées. Ce n'est pas des BeauxArts, c'est de l'art qui concerne la vie quotidienne ${ }^{4}$.»

2 Ce que nous observons n'est pas un collage à proprement parler mais la couverture d'un magazine, la livraison du mois de mai 1957 de la revue d'architecture anglaise Architectural Review. Il s'agit d'un numéro spécial intitulé « Machine Made America ». À l'intérieur, cette étrange figure est décrite comme «constituée de fragments caractéristiques du complexe culturel qu'elle symbolise: l'Amérique fabriquée à la machine ${ }^{5}$ ». En effet, ces images qui proviennent de magazines américains ont été collectées ${ }^{6}$ par John McHale lorsqu'il a quitté Londres pour l'Université de Yale afin d'y suivre l'enseignement de l'artiste et ancien maître du Bauhaus Josef Albers, durant l'année universitaire 1955-1956. Les magazines de grande distribution bénéficiaient d'une part de procédés d'impression très avancés par rapport à ceux utilisés dans la Grande-Bretagne d'après-guerre en difficile reconstruction, et regorgeaient d'autre part d'images d'opulence de la société de consommation américaine ${ }^{7}$ : la qualité des images y était, à cet égard, exceptionnelle. McHale les a utilisées en fragments directement dans ses collages et en a également fait parvenir en grande quantité à Londres, où ce matériel est venu alimenter l'imaginaire des différents artistes et théoriciens constituant son cercle intellectuel d'alors.

Ce cercle, actif autour de l'Institut d'Art Contemporain (ICA), porte le nom d'Independent Group. Ses "membres », dont la liste ne peut être clairement définie ${ }^{8}$, formaient simplement un regroupement informel de jeunes personnes issues de différents horizons. Réunis à Londres au milieu des années 1950, ils se retrouvaient pour échanger, notamment au sujet des médias de masse et des nouvelles technologies, dans une dynamique qui s'apparentait plutôt à une activité de recherche qu'à la formation d'un mouvement artistique à proprement parler. Pourtant, la nourriture visuelle mise à disposition par John McHale depuis les États-Unis ${ }^{9}$ est tenue pour avoir donné naissance à l'un des mouvements majeurs du $\mathrm{xx}^{\mathrm{e}}$ siècle, le Pop Art, dont l'Independent Group a souvent souffert d'être trop rapidement considéré comme simple initiateur. L'utilisation du matériel fourni par John McHale en 1956 par Richard Hamilton pour la réalisation du collage Just what is it that makes today's homes so different, so appealing? ${ }^{10}$, tenu pour être la première œuvre du Pop Art, a en effet très lourdement pesé sur l'historiographie et favorisé une approche " pop », en partie réductrice, qui n'a pas toujours permis de tenir compte de la spécificité ni de la diversité des réflexions ayant eu lieu au sein du groupe ${ }^{11}$.

Dans la période d'après-guerre, leurs discussions ont permis une nouvelle appréhension de la culture populaire contemporaine, ce qui explique leur intérêt tout particulier pour le foisonnement des productions américaines - ce territoire à la fois lointain par la géographie et proche par la langue, royaume du développement technologique et de la société de consommation. Cette figure inventée par McHale en est une incarnation. À l'intérieur de la revue dont elle constitue la couverture, on peut lire que les éléments de sa composition proviennent de «l'un des miroirs flatteurs préférés de l'Amérique - les illustrations en couleurs de magazines - reflétant l'univers du steak infra-cuit ${ }^{12}$, du gâteau pré-fait, des cuisines de rêve, des voitures de rêve, des machines-outils, des Robots Marie, de l'autoroute, des zones portuaires, du téléscripteur, des bougies d'allumage et de l'électronique ${ }^{13}$ ». Les objets de ces univers constituent, ensemble, cette « Amérique fabriquée à la machine » qui donnera son nom au collage Machine Made, America (1956). 
5 Pour comprendre ce titre, qui est donc à la fois celui du collage et celui du numéro spécial de Architectural Review, il faut revenir en 1950 : en décembre, la revue consacre une livraison aux États-Unis sous le titre de "Man Made America». Au fil des pages, dans une attitude antiaméricaniste assez répandue dans l'Angleterre d'alors, s'y forme l'argumentaire d'une condamnation du modèle consumériste et matérialiste américain et plus spécifiquement du modèle urbain décrit comme un environnement chaotique, plein de laissez-faire.

6 Sept ans plus tard, avec le numéro spécial « Machine Made America », un renversement du discours critique envers les États-Unis s'effectue. L'avant-propos explique ainsi que si la publication de 1950 livrait un compte rendu de la misère visuelle et du fiasco résultant des initiatives urbaines et architecturales américaines, ce numéro est celui d'une success story, contant "comment l'Amérique vient donner une plus-value aux qualités préexistantes de l'architecture moderne ${ }^{14} »$. Au même moment, de l'autre côté de l'Atlantique, le magazine américain LIFE revient justement dans son éditorial sur une formule tranchante du numéro "Man Made America » de 1950 qui décrivait alors le «bazar qu'est l'Amérique fabriquée par l'homme ${ }^{15}$ »:

En effet, il y a un véritable bazar fabriqué par l'homme autour de nous. Ce pays est effectivement une immense fourmilière grouillante (et pleine de fougue). Mais où dans l'histoire a-t-on déjà pu voir un tel flux tumultueux d'hommes, de biens de consommation et de machines, baignant dans une telle liberté, le tout dans les limites de l'état de droit et du respect de l'ordre public? Une société capable de générer cela est une société prête à se confronter à son présent et aux nouveaux défis qui s'offrent à elle - créer la première société moderne, technologique, humaine, prospère et digne de respect religieux - pour véritablement faire de notre pays l'Amérique, la Magnifique. ${ }^{16}$

Le bouillonnement et l'enthousiasme de ce texte, publié dans un magazine de diffusion de masse typique de ceux auxquels s'intéressait McHale, donnent une idée de l'atmosphère dans laquelle l'artiste a baigné tout au long de son séjour aux États-Unis. On sait d'ailleurs que LIFE constituait l'une de ses sources de collecte pour les images utilisées dans ses collages ${ }^{17}$.

\section{Robot anthropomorphe}

7 L'œuvre Machine Made, America est figurative au sens dans lequel l'entend l'anthropologue Philippe Descola: elle évoque un prototype «en jouant sur une ressemblance directe de type mimétique ${ }^{18} »$. La présence d'éléments évoquant des yeux, le dosage de la distance entre eux et d'autres éléments faisant allusion à un nez nous suffit à reconnaître ici un visage, selon le mécanisme à l'œuvre dans les peintures d'Arcimboldo. Mais cette fois, l'être hybride émergeant de l'image n'est pas humain : il s'agit plutôt d'un robot anthropomorphe ${ }^{19}$. Reyner Banham, un autre membre de l'Independent Group, insiste sur l'ambiguïté de cette hybridation: «Cette figure à la tête en forme de dôme, émaillée de preuves enchevêtrées des prouesses des États-Unis en matière de technologie, de moyens de communication et d'opulence - composée d'éléments imprévisiblement mécaniques ou végétaux, animaux ou arithmétiques était, néanmoins, délibérément et incontestablement humanoïde ${ }^{20} »$. On pourrait croire y reconnaitre Robbie le Robot, l'un des personnages centraux du film de science-fiction Planète Interdite $e^{21}$ (fig. 3) sorti au cinéma l'année de la création de ce collage, en 1956. 
Fig. 3

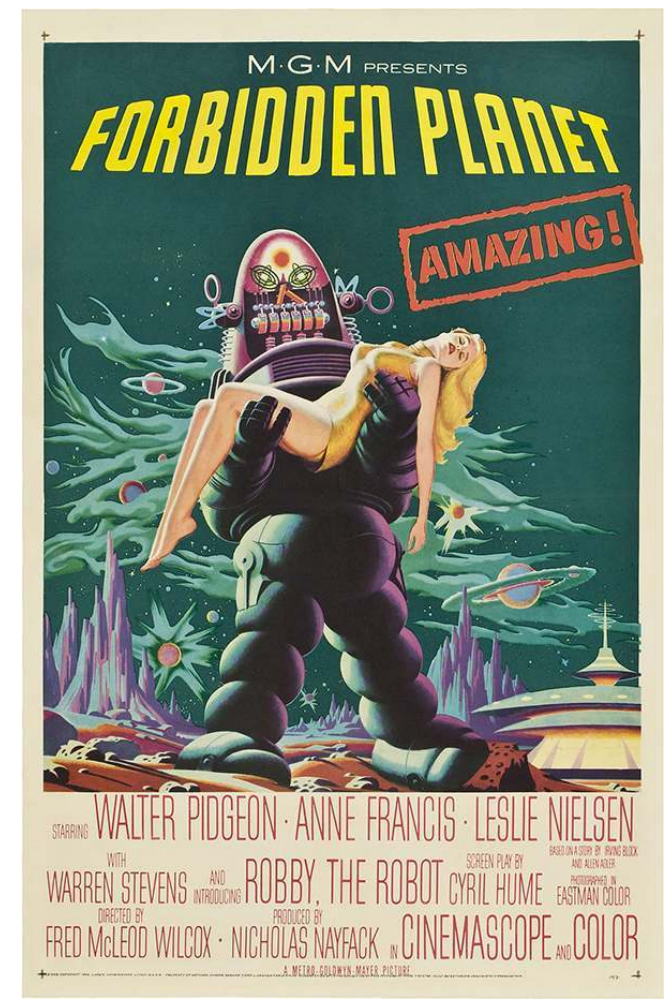

Affiche du film Forbidden Planet, Metro-Goldwyn-Mayer Studios Inc., production : Nicholas Nayfack, réalisation : Fred M. Wilcox

(c) Turner Entreprises, Inc., Culver City, Calif.

Le rapprochement entre les deux figures est d'autant plus manifeste que Robbie est devenu, de façon fortuite et par l'intermédiaire de l'Independent Group, l'un des acteurs de ce que l'on tient pour « l'acte de naissance » du Pop Art à Londres. Il s'agit de l'exposition This is Tomorrow ${ }^{22}$, qui a lieu elle aussi en 1956 et qui compte parmi ses participants de nombreux membres du groupe, dont John McHale et Richard Hamilton. Ensemble, ils mettent au point une section de l'exposition sous la forme d'un environnement immersif dénommé Fun House ${ }^{23}$. Ils cherchent à y retranscrire les différents stimuli de la vie quotidienne d'alors et, notamment, l'atmosphère tumultueuse de la culture populaire. C'est à ce titre qu'ils s'approprient l'image de Robbie le Robot. Celui-ci cumule les qualités recherchées : éminemment contemporain, il est issu de la science-fiction - genre cinématographique associé à la culture populaire - et qui plus est de la science-fiction américaine. Robbie est, dans le film, une création du professeur Morbius, échoué sur la planète Altaïr IV, seul survivant de l'équipage d'un vaisseau d'exploration avec sa femme et sa fille, Altaïra. Notons que, malgré l'affiche trompeuse, Robbie est plutôt présenté, au sein de la narration, comme un outil domestique que comme une conséquence dangereuse des avancées robotiques. 
Fig. 4

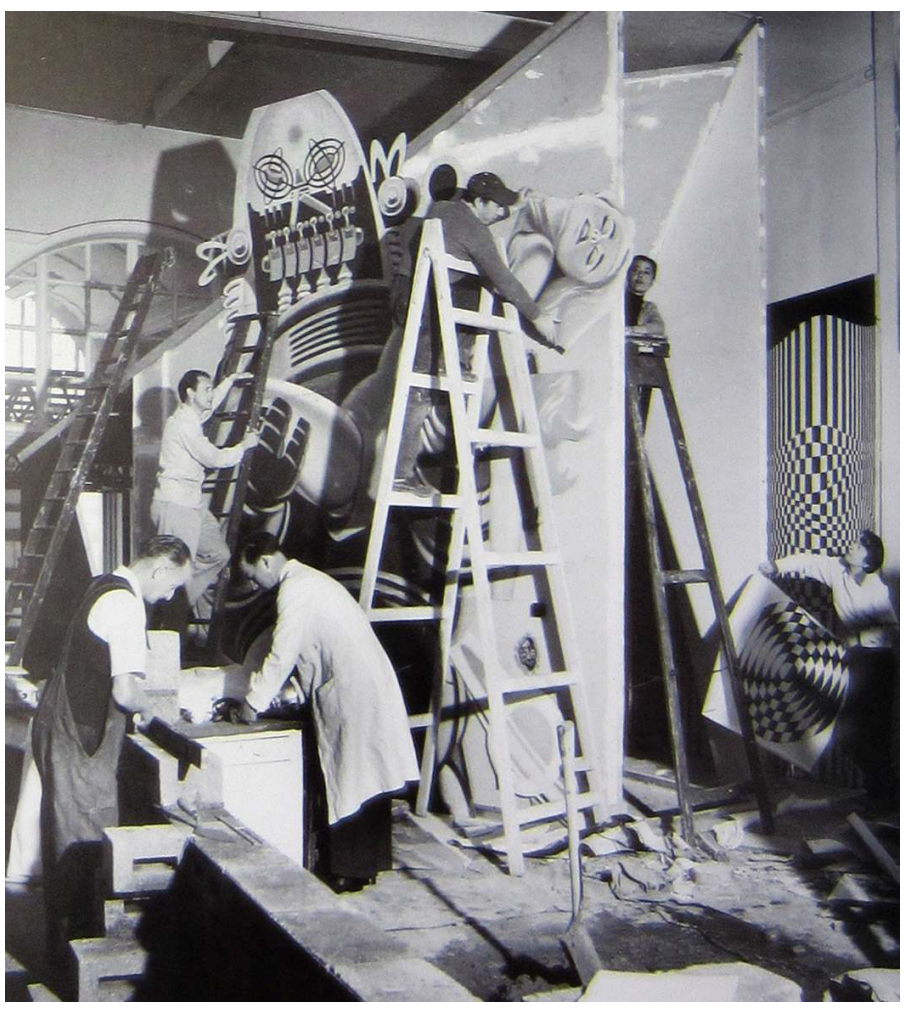

Les membres du groupe 2 et leur entourage s'affairant à l'installation de la Fun House pour l'exposition This is Tomorrow, 1956, Whitechapel Gallery, Londres. Sur les échelles, de gauche à droite : John McHale, Richard Hamilton et Magda Cordell. The Architects' Jounal, n 3207, août 1956, p. 219.

(c) Architect's Journal, Londres

Sur les murs de la Fun House s'étend une immense reproduction, extraite de l'affiche du film (fig. 4), sur laquelle Robbie porte dans ses bras l'héroïne Altaïra. Mais McHale et Hamilton ne s'arrêtent pas là : ils font intervenir Robbie le Robot « en personne » lors du vernissage de l'exposition. Pour cela, ils empruntent une mascotte qui était utilisée pour la promotion du film et, lors de la soirée d'ouverture, un membre de l'ICA se glisse dans le costume du personnage fictionnel. Il déambule parmi le public avant de prononcer un discours écrit par Lawrence Alloway, l'un des membres les plus éminents de l'Independent Group (fig. 5). 
Fig. 5

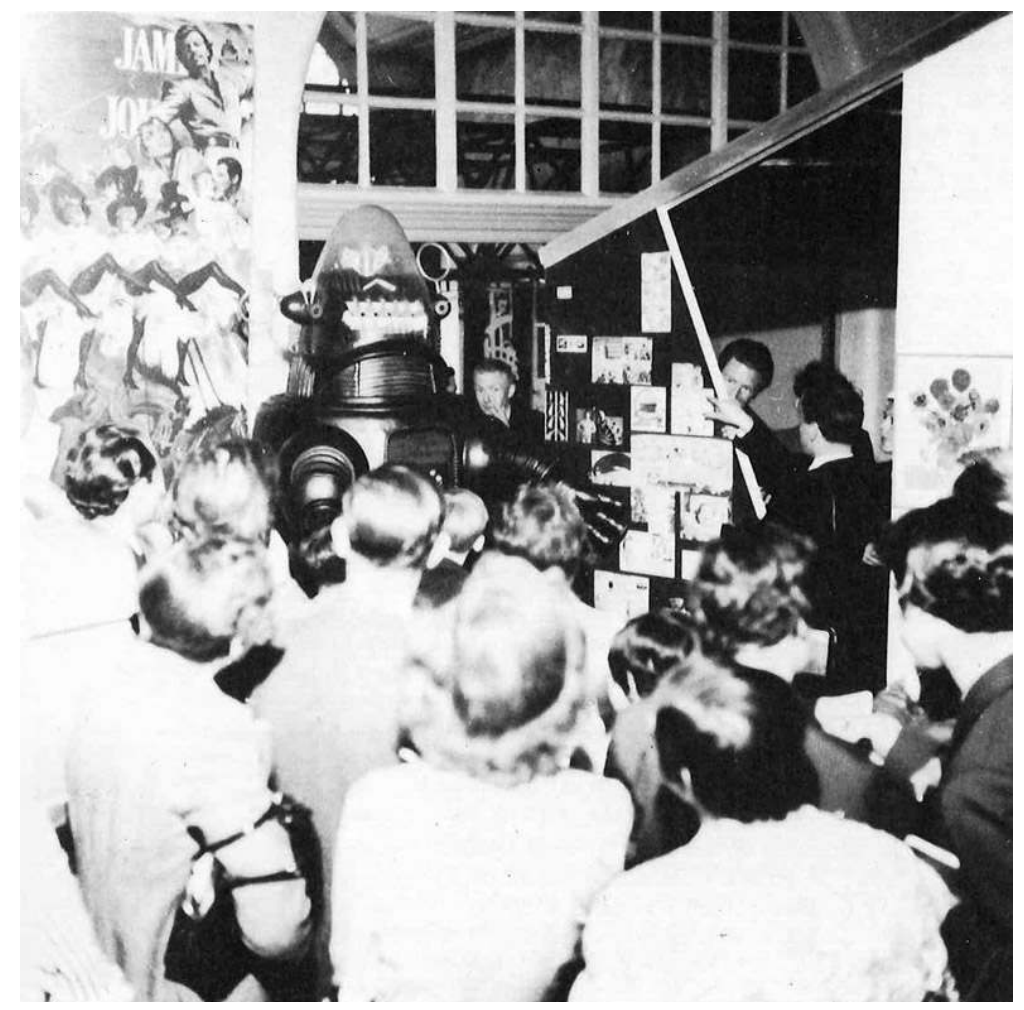

La mascotte de Robbie le Robot, personnage du film Forbidden Planet, lors du vernissage de l'exposition This is Tomorrow, 1956, Whitechapel Gallery, Londres. En arrière-plan : Lawrence Alloway (derrière Robbie), John McHale (dans la Fun House).

(c) Richard Hamilton Estate

Dans un article d'époque ${ }^{24}$, Alloway décrit l'apparition de Robbie le Robot au vernissage de This is Tomorrow comme représentative de l'art de l'âge de la machine dans ses formes les plus éclatantes. Il livre ensuite une brève analyse de la place des robots dans l'art populaire, qui, selon lui, symbolise tantôt la subordination de la machine (notamment dans la sphère domestique), tantôt la prise de pouvoir du robot sur l'être humain. Mais il note que, dans tous les cas, le robot conserve une ressemblance avec l'image humaine à partir de laquelle il est systématiquement façonné25. Créer le robot à son image est pour l'homme une manière d'atténuer l'étrangeté de la machine. Reyner Banham reviendra ultérieurement sur cette dialectique du rapport entre la figure du robot et l'image humaine à travers un commentaire de Machine Made, America. Il écrira, à propos de ce collage :

Sa forme compacte, frontale et symétrique, rappelait les fréquents débats qui se tenaient entre les membres de l'Independent Group sur ce que devait être le degré d'humanité approprié des robots. Faire un robot à cette époque revenait à affirmer sa foi en l'homme et en l'humanité - sinon à en faire profession - selon une tradition qui remontait de Frankenstein aux automates du Siècle des Lumières, en passant par le Golem et la «ressemblance parlante ${ }^{26}$ de la sculpture de la Renaissance jusqu'à la légende de Pygmalion, et peut-être même jusqu'aux plus anciennes peintures rupestres de figures humaines sous la forme d'animaux totémiques producteurs de denrées alimentaires - car Robby [sic.] le Robot, dans le film Planète interdite, n'était-il pas producteur de denrées alimentaires sous une forme quasi humaine $?^{27}$

En effet, Robbie possède la capacité de reproduire les molécules de toutes les substances qu'il ingère, y compris - dans une scène restée célèbre - deux cents litres de 
bourbon "Kansas City", pour le plus grand bonheur des humains ${ }^{28}$ (fig. 6). Banham remonte à la préhistoire pour évoquer cette tradition de figures totémiques douées de caractères surhumains, passant par le Golem dont l'image de créature d'argile tend d'ailleurs, dans les comics américains du $\mathrm{xx}^{\mathrm{e}}$ siècle, à être remplacée par une image de robot $^{29}$.

Fig. 6

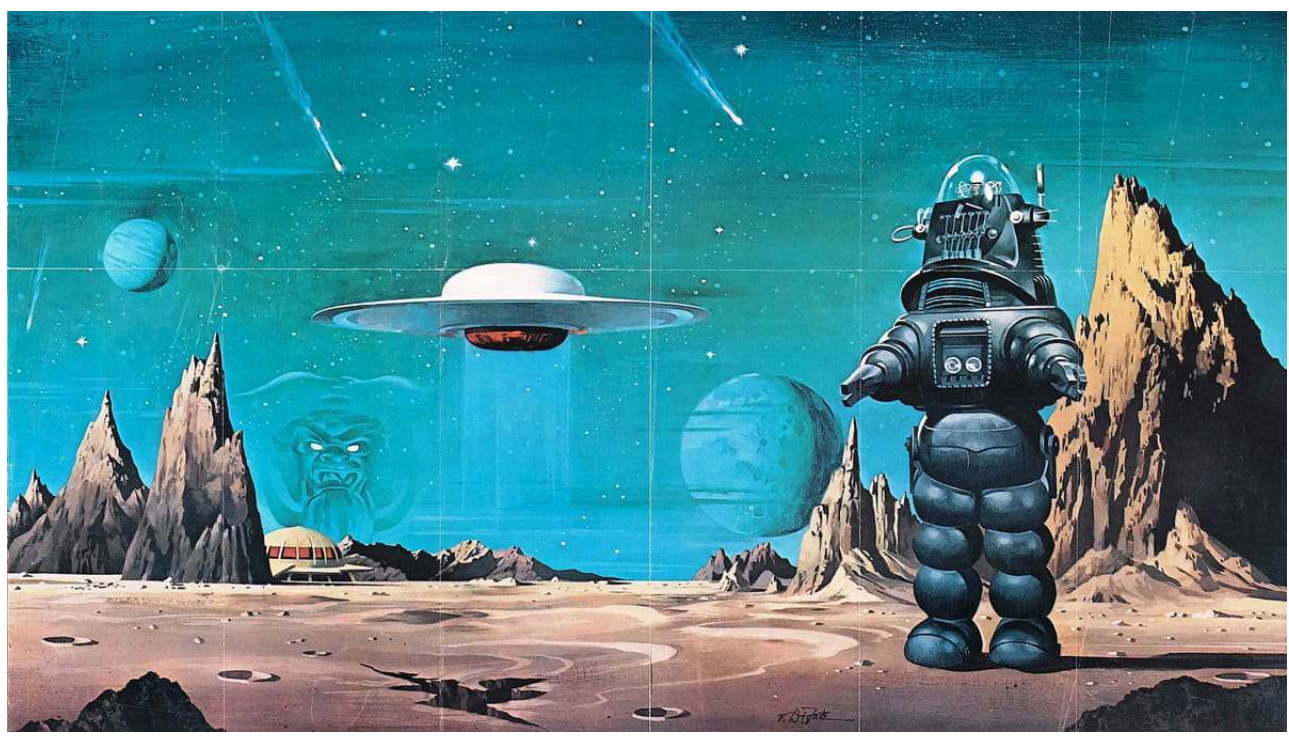

Planète Interdite (1956)

(c) iMdb

Ce rapport mythique que Banham crée avec la figure de Machine Made, America était déjà mis en avant à l'intérieur de Architectural Review : un commentaire s'arrêtait alors sur l'image de levier de vitesse automobile que l'on peut distinguer sur le thorax de la figure, le qualifiant de "tétragramme du pouvoir - point mort, marche avant, première, marche arrière - gravé sur son cœur $»^{30}$. À travers ces mots est convoquée une divinité de la mécanique amenée à remplacer le Tétragramme hébraïque, YHWH.

\section{Icônes jetables}

10 McHale, qui a beaucoup écrit parallèlement à sa production plastique, emploie luimême un vocabulaire religieux dans un article publié quelques années plus tard, en 1959, sous le titre "The Expendable Ikon » [L’Icône jetable] ${ }^{31}$. Il y étudie les éléments des médias de masse qui deviennent, au fil de leur diffusion, ce qu'il considère comme des «icônes » - mais temporairement, avant d'être remplacées par d'autres car dans ces médias, écrit-il, «la seule réelle constante est le changement ». Il fait référence à des objets élevés à un "niveau quasi totémique» et parle d'un processus de "ritualisation", fruit de la répétition de la présentation de l'image qui devient, dès lors, une référence, un symbole, un mythe collectif. Par ailleurs, McHale prend soin de souligner le rôle traditionnellement attribué aux Beaux-Arts dans l'organisation et la régulation de notre rapport au monde, justifiant ainsi son recours au terme d'icône :

L'accent porté sur le terme d'«icône » découle d'une préoccupation pour ce qui est la fonction même de la quasi-totalité du champ de la communication visuelle. Audelà de la transmission de simples messages sur l'organisation de la réalité du 
monde actuel tel qu'il est perçu, on trouve un niveau de communication plus complexe passant par des signes, des symboles ou des "images chocs ", véhiculant des messages sur la condition de l'homme dans son environnement considéré dans sa totalité. Historiquement, cette dernière fonction relevait des beaux-arts. Elle impliquait toute une réserve d'«images exploitables", de "configurations de l'expérience humaine», de "constructions symboliques de la réalité » qui permettaient à l'homme de se situer dans son environnement et de le maîtriser qu'il s'agisse d'un univers extérieur ou intérieur - même si cette dichotomie est fragile. De telles constructions peuvent être des totems, des masques, une danse rituelle, un poème ou une cathédrale.

En général, aux époques qui nous sont antérieures, les icônes avaient tendance à durer relativement longtemps; elles relevaient d'une cosmogonie donnée en lien avec un dogme monolithique et, quoique répondant à des contextes régionaux, elles se réclamaient d'une universalité qui était la condition première de leur acceptation $^{32}$.

Par contraste, les icônes contemporaines évoquées par McHale dans cet article sont éphémères, jetables. Puisées dans l'environnement quotidien, elles sont utilisées puis, une fois leur contenu symbolique consommé, remplacées par d'autres icônes assurant une fonction similaire.

Au même moment, en France, Roland Barthes se consacre à une réflexion du même ordre sur le processus de fabrication des mythes contemporains et publie ses Mythologies, fragments de réflexions menées au fil des jours à partir de ses rencontres avec des éléments du quotidien. Il consacre d'ailleurs une section au bifteck, celui-là même dont McHale avait fait une épaule pour sa figure Machine Made, America. Roland Barthes s'intéresse plutôt au bifteck français, bien qu'il le reconnaisse "circonscrit, il est vrai, aujourd'hui par l'invasion des steaks américains ». Selon lui, "c'est la nourriture à la fois expéditive et dense, il accomplit le meilleur rapport possible entre l'économie et l'efficacité, la mythologie et la plasticité de sa consommation ${ }^{33}$ ». Chaque élément utilisé dans le collage de McHale pourrait être décrypté de cette manière, en fonction de la mythologie à laquelle son image se réfère. Car c'est bien l'image d'un objet que McHale convoque et non l'objet lui-même, la reproduction de cet objet par les médias de masse constituant l'essence même de son contenu symbolique. L'artiste écrit ailleurs que «la photographie et les techniques modernes d'impression produisent une nouvelle image visuelle, dont l'“expérience" est, en réalité, assez distante de celle engendrée par l'artefact original ${ }^{34} »$.

11 De la même manière, ce collage n'est plus qu'une image de lui-même. Conçu dans le but d'être reproduit en couverture d'une revue, personne ne s'est soucié d'en conserver l'original $^{35}$. Il existe bien une reproduction photographique, signée de la main de l'artiste, mais ici encore, elle ne fait office d' « œuvre » qu'en tant qu'image de l'original perdu (et pas en tant que photographie). En cela, Machine Made, America est bien une œuvre Pop : comme l'écrit l'historien de l'art Alex Kitnick, «le Pop a tracé le virage menant d'un monde d'objets à un univers d'images, qui, par la force des choses, a également engendré de nouvelles formes de subjectivité ${ }^{36}$. " Ici, la création originale s'est dissoute dans son caractère transitoire, éphémère, consommable - seule subsiste son image qui, reproduite telle qu'elle l'est en illustration de cet article, continue de faire exister l'œuvre. 


\section{NOTES}

1. Né en 1922 à Glasgow, mort en 1978 à Houston, Texas, John McHale a vécu à Londres à partir de la fin des années 1940 puis dans diverses villes des États-Unis à partir de 1962 et jusqu'à sa mort précoce. Pour des détails sur ses diverses activités voir Juliette Bessette, « John McHale : de l'art du collage à la pensée prospective ", Les Cahiers du musée national d'Art moderne, n ${ }^{\circ} 140$, été 2017, p. 34-58.

2. Lawrence Alloway, "Paolozzi and the Comedy of Waste", Cimaise, octobre-décembre 1960, p. 120, consulté dans Nigel Whiteley, Art and Pluralism. Lawrence Alloway's Cultural Criticism, Liverpool, Liverpool University Press, 2012, p. 100. «[...] the throwaway materials of our culture in impression, [and thus] resemble ourselves as consumers. » En cela, écrit Alloway, la figure acquiert une valeur symbolique car elle ressemble à ses contemporains «en tant que consommateurs. » Sauf indication contraire, toutes les traductions sont de l'auteure.

3. Lawrence Alloway, «The Development of British Pop Art» dans Lucy Lippard (dir.), Pop Art, Londres, Thames \& Hudson, 1966, p. 27-68, traduction française : «Le Développement du Pop Art anglais ", Le Pop Art, Paris, Thames \& Hudson, 1996, p. 27-67. Alloway fait notamment référence à la série des Heads [Têtes], 1954-1958.

4. Robert Freeman, "The Human Image ", Lady Clare, juin 1959, p. 8. "These images are defined by what they consume [...] Hence they are as expendable as the consumer materials which go into their making. Not fine art, but art related to everyday life. »

5. Ian McCallum (éd), Architectural Review, numéro spécial « Machine Made America.», Londres, mai 1957, p. 393. «The cover personage [...] was assembled from typical fragments of the cultural complex that he also symbolizes; Machine Made America. »

6. Les sources des fragments n'ayant pas été intégralement retracées, il est possible que certains d'entre eux proviennent de magazines publiés dans d'autres pays, notamment en Angleterre. Nous ne savons pas si McHale a assemblé ces images sur place ou s'il les a mises dans ses bagages lorsqu'il a quitté les États-Unis en mai 1956 pour réaliser cette composition une fois de retour à Londres.

7. À la suite de la guerre, le rationnement n'a pris fin en Angleterre qu'en 1954.

8. Les personnes gravitant autour de l'Independent Group (artistes, architectes, théoriciens, etc.) vont et viennent mais citons, entre autres, Reyner Banham, Eduardo Paolozzi, Richard Hamilton, Lawrence Alloway, Alison Smithson, Peter Smithson, William Turnbull, Toni del Renzio, Nigel Henderson, Magda Cordell, James Stirling, Colin St John Wilson, John Voelcker, Frank Cordell, Edward Wright et, donc, John McHale.

9. Magda Cordell, la compagne de John McHale, lui rend visite et rentre à Londres en mars 1956 avec, dans ses bagages, un grand coffre rempli de magazines et de coupures. McHale rapportera d'autres éléments à son retour au mois de mai.

10. Richard Hamilton, Just what is it that makes today's homes so different, so appealing? 1956, collage, $26 \times 25 \mathrm{~cm}$, Kunsthalle, Tübingen. Pour la genèse du collage, voir John-Paul Stonard, «Pop in the Age of Boom: Richard Hamilton's 'Just what is it that makes today's homes so different, so appealing? ", The Burlington Magazine, CXLIX, septembre 2007, vol. 149, no. 1254, p. 607-620.

11. Sur l'Independent Group, voir le catalogue de référence : The Independent Group: Postwar Britain and the Aesthetics of Plenty, sous la direction de David Robbins, Darmouth College, Hood Museum of Art; Londres, Institut d'art contemporain; Los Angeles, The Museum of Contemporary Art ; Berkeley, University of California Art Museum, Cambridge, The MIT Press, 1990, ou plus récemment: Anne Massey, Out of the Ivory Tower. The Independent Group and Popular Culture, Manchester, Manchester University Press, 2013. 
12. Note de l'auteure: l'expression se réfère visiblement à la technologie de la cuisson « infrarouge ».

13. Ian McCallum (éd), Architectural Review, op. cit. note 5, p. 393. "The source of material was one of America's favourite flattering mirrors, coloured magazine illustrations, and reflects a world of infra-grilled steak, pre-mixed cake, dream-kitchens, dream-cars, machine-tools, power-mixers, parkways, harbours, ticker-tape, spark-plugs and electronics. »

14. Ian McCallum (éd), Architectural Review, op. cit. note 5, p. 393. « The previous special number of the ARCHITECTURAL REVIEW concerned with the USA was a record of sprawl and visual squalor (Man Made America, Dec., 1950), the record of a failure. The present issue, compiled, written and annotated by the executive Editor, Ian McCallum, is a success story - the story of how America is adding sheer quantity to the pre-existing qualities of modern architecture. "

15. Architectural Review, numéro special « Man Made America.», Londres, décembre 1950. «[...] the mess that is man-made America ».

16. Editorial «America - the Beautiful?», LIFE, 3 juin 1957, p. 34. «Indeed there is much manmade mess around us. It is a great swarming ant heap of a country (with lots of pizazz) [sic.]. But where before in history has there ever been such a turbulent flow of men and goods and machines, with so much liberty, within the disciplines of law and order? A society capable of creating this will be able to meet its present and newest challenge - to create the first modern, technological, humane, prosperous and reverend society - to make our land truly America the Beautiful. »

17. Voir les "Palettes", coupures de magazines collectées par McHale et conservée au Yale Center for British Art [McHale Archive. Collection, Rare Books and Manuscripts].

18. Philippe Descola, texte de présentation du cours « Modalités de la figuration» (2005-2006), chaire d'Anthropologie de la nature, collège de France: https://www.college-de-france.fr/ media/philippe-descola/UPL35669_descola_cours0506.pdf [08.10.18]. Il entend la figuration comme une opération visant à donner à «un objet matériel quelconque [...] un potentiel d'évocation iconique d'un prototype réel ou imaginaire qu'il dénote de façon indicielle (par délégation d'intentionnalité) en jouant sur une ressemblance directe de type mimétique ou sur tout autre type de motivation identifiable de façon médiate ou immédiate. »

19. McHale a réalisé une seconde œuvre du même type l'année suivante, également intitulée Machine Made, America. Le collage original est conservé dans un fond actuellement inaccessible, c'est pourquoi il n'est pas reproduit dans cet article (John McHale, Machine Made, America, 1957, collage sur papier, $56 \times 71 \mathrm{~cm}$, The John McHale and Magda McHale Archives Foundation, Buffalo, New York).

20. Reyner Banham in The Expendable Ikon: Works by John McHale, Albright-Knox Art Gallery, Buffalo, 12 mai-8 juillet 1984, cat. sous la dir. de Charlotta Kotik, Buffalo, Manhardt-Alexander Inc., 1984, p. 40. « That dome-headed figure, encrusted with overlapping evidences of the United States prowess in technology, communications and affluence, unpredictably mechanistic or vegetative, animal or arithmetic in its elements, was, nevertheless, recognizably and deliberately humanoid. » Reyner Banham est critique et théoricien de l'architecture.

21. Forbidden Planet, 1956, Metro-Goldwyn-Mayer Studios Inc., production: Nicholas Nayfack, réalisation : Fred M. Wilcox.

22. This is Tomorrow, 9 août-9 septembre 1956, Whitechapel Gallery, Londres, cat. sous la dir. de Theo Crosby, Londres, 1956. Le catalogue a été réédité par la Whitechapel Gallery à l'occasion de la reconstitution d'une partie de l'exposition à l'ICA en 1990.

23. John McHale et Richard Hamilton sont réunis, avec l'architecte John Voelcker qui met au point la structure de la Fun House, au sein de la section numéro 2 (l'exposition étant divisée en 12 sections). Ils sont largement aidés d'autres membres de l'IG qui ne participent pas officiellement à l'exposition This is Tomorrow : Terry Hamilton, Frank Cordell et Magda Cordell. 
24. Lawrence Alloway, "The Robot \& the Arts ", Art News and Review, vol. 1, n 16, $1^{\text {er }}$ septembre 1956, p. 1, p. 6.

25. Dès l'origine du mot " robot ", qui apparaît pour la première fois dans une pièce de sciencefiction de l'écrivain tchèque Karel Čapek (R.U.R., Rossumovi univerzální roboti, 1920), le robot est une machine, un organisme artificiel dont l'apparence ne permet pas de le différencier de l'être humain.

26. Note de l'auteure: les guillemets entourant «speaking likeness" dans le texte original renvoient à l'expression popularisée par Rudolf Wittkower (notamment dans son Bernini's bust of Louis XIV, Londres, Oxford University Press, 1951).

27. Reyner Banham dans The Expendable Ikon: Works by John McHale, op. cit. note 20, p. 40. «Its compact and symmetrically frontal form recalled the frequent argumentation among Independent Group members about the proper degrees of humanity robots. To make a robot in those times was to make a statement about, if not a proclamation of faith in, humanity and the humane tradition, that went back through Frankenstein and the automata of the Age of Enlightenment, through the Golem and the "speaking likenesses" of the Renaissance sculpture to the legend of Pygmalion, perhaps even to the earliest cave-paintings of men as food-producing totemic animals - for was not Robby the Robot in the film Forbidden Planet a food-producer in almost-human form?»

28. Extrait du film : < http://www.criticalcommons.org/Members/rsmith/clips/from-forbiddenplanet-old-customs/view >

29. Cat. d'exp. Golem! Avatars d'une légende d'argile, sous la direction de Ada Akerman, musée d'art et d'histoire du Judaïsme, Paris, 8 mars-16 juillet 2016, Paris mahJ-Hazan, 2016.

30. Ian McCallum (éd), Architectural Review, op. cit. note 5, p. 393. «[...] the tetragram of power Neutral, Drive, Low, Reverse - graven on his heart [...]».

31. John McHale, "The Expendable Ikon », Architectural Design, vol. 29, février 1959, p. 82-83 et «The Expendable Ikon II ", Architectural Design, vol.29, février 1959, p. 116-117, traduction française : John McHale, «L'icône jetable » (1959), traduit par Juliette Bessette et Hervé Vanel, Les Cahiers du musée national d'art moderne, $\mathrm{n}^{\circ}$ 140, été 2017, p. 59-67.

32. John McHale, "The Expendable Ikon», Architectural Design, vol.29, février 1959, p. 82, traduction Ibid.

33. Roland Barthes, Mythologies, Paris, Seuil, 1957, p. 85.

34. John McHale, "The Fine Arts in the Mass Media ", Cambridge Opinion, $n^{\circ} 17,1959$, p. 29. «Photography and modern writing techniques produce a new visual image, the 'experience' of which is, in reality, at some remove from that engendered by the original artefact [...]. »

35. La revue Architectural Review affirme ne pas avoir gardé trace du collage original après l'utilisation de son image pour la livraison de mai 1957. La reproduction photographique évoquée est conservée par The John McHale and Magda McHale Archives Foundation, Buffalo, New York [fond inaccessible], 58,4 $\times 43,2 \mathrm{~cm}$.

36. Alex Kitnick, «Pop Pyramid», dans Alex Kitnick on Derek Boshier at Thomas Solomon Gallery, Los Angeles, Texte Zur Kunst, no 23, juin 2013, p. 221. «What Pop did was trace the shift from a world of objects to a universe of images, which by necessity gave rise to new forms of subjecthood as well. » 


\section{RÉSUMÉS}

En 1956, l'artiste John McHale rentre à Londres après une année passée à l'Université de Yale. Des États-Unis, il rapporte des dizaines de magazines de diffusion de masse dont les pages abondent d'images de biens de consommation et d'objets de culture populaire. A partir de ce matériau, il produira de nombreux collages, détournant le sens iconographique de fragments choisis en les réemployant au sein de nouvelles compositions. Dans le collage intitulé Machine Made, America [L'Amérique, fabriquée à la machine] apparaît une figure intrigante évoquant un robot, d'un impact visuel «pop» très puissant. Sa portée symbolique s'appréhende dans un contexte historique et culturel spécifique, celui de l'Angleterre en reconstruction des années d'aprèsguerre.

In 1956 the artist John McHale returned to London after spending a year at Yale University. From the United States, he brought back dozens of mass-market magazines whose pages were full of images of consumer goods and objects of popular culture. From this material, he produced several collages, twisting the iconographical sense of the fragments chosen by reusing them in new compositions. In the collage entitled Machine Made, America appeared an intriguing figure evoking a robot, with a very powerful "Pop" visual impact. Its symbolic value is grasped in a specific historical and cultural context, that of the United Kingdom in reconstruction during the post-war years.

\section{INDEX}

Keywords : Independent Group, Pop Art, collage, robot, popular culture, science fiction, visual culture, icon, hybridisation, mechanomorphism

Mots-clés : Independent Group, Pop Art, collage, robot, culture populaire, science-fiction, culture visuelle, icône, hybridation, mécanomorphisme

\section{AUTEUR}

\section{JULIETTE BESSETTE}

Diplômée de l'École du Louvre, Juliette Bessette y a initié son travail de recherche sur John McHale qu'elle prolonge actuellement dans le cadre d'un doctorat sous la direction d'Arnauld Pierre (Sorbonne Universités, Centre André Chastel). Elle a été Visiting Scholar à l'Université de Yale, où sont conservées une partie des œuvres et des archives de McHale. Ses recherches portent sur l'impact des développements technoscientifiques de l'après Seconde guerre mondiale sur la création artistique d'alors.

***

A graduate of the École du Louvre, Juliette began her research on John McHale there, which she is extending as part of a doctorate under the supervision of Arnauld Pierre (Sorbonne Universités, Centre André Chastel). She was Visiting Scholar at Yale University, where part of McHale's works and archives are kept. Her research focuses on the impact of the technical and scientific developments of post-Second World War period on the artistic creation of the time. 